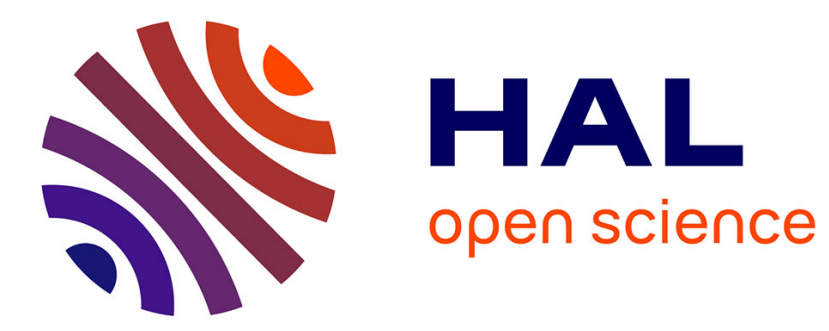

\title{
Performance analysis of the matched-pulse-based fault detection
}

\author{
Layane Abboud, Andrea Cozza, Lionel Pichon
}

\section{To cite this version:}

Layane Abboud, Andrea Cozza, Lionel Pichon. Performance analysis of the matched-pulse-based fault detection. Complex Systems Design \& Management (CSDM 2010), Oct 2010, Paris, France. pp.XX. hal-00485669

\section{HAL Id: hal-00485669 \\ https://hal.science/hal-00485669}

Submitted on 18 Nov 2010

HAL is a multi-disciplinary open access archive for the deposit and dissemination of scientific research documents, whether they are published or not. The documents may come from teaching and research institutions in France or abroad, or from public or private research centers.
L'archive ouverte pluridisciplinaire HAL, est destinée au dépôt et à la diffusion de documents scientifiques de niveau recherche, publiés ou non, émanant des établissements d'enseignement et de recherche français ou étrangers, des laboratoires publics ou privés. 


\section{Performance Analysis of the Matched-Pulse-Based Fault Detection}

Layane Abboud, Andrea Cozza and Lionel Pichon

\section{Introduction}

The problem of fault detection and location in wire networks has gained an increasing importance in the last few years [1]. Wired networks are found in all modern systems, and are used to transmit different signals (control, alarm, etc.). That is why the issue of safe and reliable wiring systems is among the primary concerns of researchers and government agencies today [2].

There are several methods for wire testing, such as visual inspection, impedance testing [1], and reflectometry methods which are widely used today to help detecting and locating wire faults. These methods send a predefined testing signal down the wire network to be examined. They include Time Domain Reflectometry (TDR), which uses a fast rise time step or pulsed signal as the testing signal, Frequency Domain Reflectometry [3] which uses multiple sinusoidal signals, sequence TDR [4] which uses pseudo noise, etc. Generally, hard faults (open and short circuits) are detectable through standard reflectometry, while soft faults (damaged insulation, etc.) are more critical to detect, especially when dealing with complex wire networks configurations.

In [5], we introduced the Matched-Pulse approach (MP), based on the properties of Time Reversal [6], as an improvement of the existing standard TDR. The MP method proposes to adapt the testing signal to the network under test, instead of using a predefined testing signal, as opposed to reflectometry methods. We have

Layane Abboud

Département de Recherche en Électromagnétisme, SUPELEC, 3 rue Joliot-Curie, 91192 Gif-surYvette, France. e-mail: Layane. Abboudesupelec.fr

Andrea Cozza

Département de Recherche en Électromagnétisme, SUPELEC, 3 rue Joliot-Curie, 91192 Gif-surYvette, France. e-mail: Andrea.Cozza@supelec.fr

Lionel Pichon

Laboratoire de Génie Électrique de Paris, LGEP - CNRS / SUPELEC - 91192 Gif-sur-Yvette,

France. e-mail: lionel.pichond lgep. supelec. fr 
shown that this method results in a higher echo energy from the fault to be detected, when compared to TDR.

In this paper, we propose a sensitivity analysis of the MP approach, i.e., to evaluate the effect of the network topology (junctions, discontinuities, etc.) on the performance of this approach when compared to standard TDR. Through topological analysis, we will illustrate the role of different network elements governing the performances of both the TDR and MP. A mathematical analysis then follows in order to compare our approach to the TDR. The whole study will allow us to predict, for a given system configuration, to which extent the MP would be more beneficial compared to TDR, and state the configurations where our approach presents major advantages.

The paper is organized as follows: first we give the general assumptions we considered in our work, and then give a brief reminder about the MP approach, followed by a topological study of the influence of the network elements on the TDR performance. A mathematical analysis following this study allows to establish a comparison criterion of the MP and TDR, and finally simulation results permit a better validation of the discussed ideas.

\section{The wire network}

In this paper, we will consider uniform lossless transmission lines. For the sake of simplicity, we also consider resistive parallel loads and do not use reactive components, so that there is no dispersion. We will also consider that the testing signal (i.e., the injected signal) is the input to the system, and the reflected voltage wave is the output. In the presence of an eventual fault, we can either analyze the reflected signal directly or take the difference of the reflected signals, both in the presence and absence of the fault, in order for the echo corresponding to the fault to be more easily detected.

In this paper, provided that the systems we are studying are linear time invariant, we will be considering the difference of the reflected signals. Let the transfer function of the reference system without the fault be noted as $H_{0}(f)$, and the one corresponding to the system with the fault noted as $H_{F}(f)$. Consequently, analyzing the difference signal is equivalent to analyzing the output of a system whose transfer function $H(f)$ is

$$
H(f)=H_{F}(f)-H_{0}(f)
$$

We will refer to this system as the difference system. 


\section{The MP approach}

Before proceeding with our topological study, we remind the basic concept of the MP approach. Instead of injecting a predefined testing signal into the network to be analyzed, as for existing reflectometry methods, the idea is to adapt the injected signal to the system, so that the energy of the echo from the fault is maximized, thus increasing its detection probability. Such a tailor-made signal, or MP, can be synthesized considering the matched filter approach used in signal processing [7]. According to this theory, a matched filter is obtained by correlating a known signal, or template, with an unknown signal to detect the presence of the template in the unknown signal. This is equivalent to convolving the unknown signal with a timereversed version of the template. The matched filter is the optimal linear filter for maximizing the signal to noise ratio (SNR) in the presence of additive white noise. To resume, in the MP approach, the testing signal is synthesized as follows: we first inject the TDR testing signal in the network under test. The received echo is next time reversed; this signal is now the MP testing signal. We clearly notice that this signal changes according to the network's architecture, as opposed to the standard TDR.

In this paper, we propose an analysis of the performance of both TDR and MP, in order to better understand and characterize this approach. Through topological analysis and a mathematical study, we will be able to understand the reason why, in some cases, the MP presents major advantages over the TDR, and predict, for a given network architecture, the performance of the TDR and MP.

\section{Topological study}

After a brief description of the general assumptions considered in this paper, along with reminding the general idea of the MP approach, we now propose to analyze the impact of different network elements on the performances of both the TDR and MP approaches. But first let us introduce the topological representation we chose to better illustrate the discussed ideas.

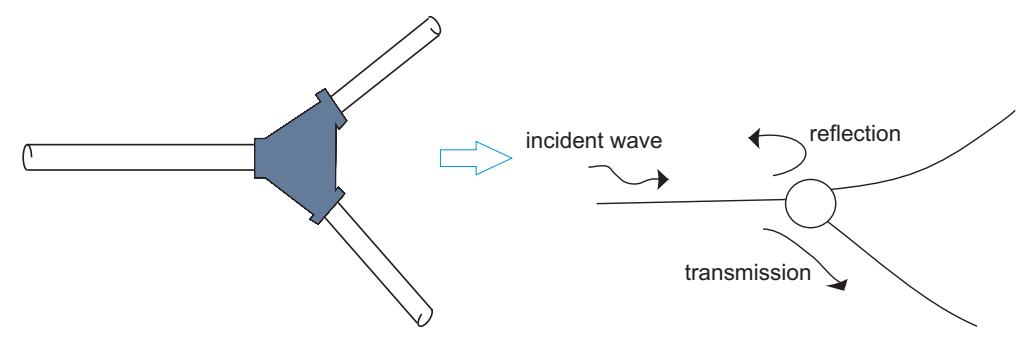

Fig. 1 An example of an equivalent topological representation. 


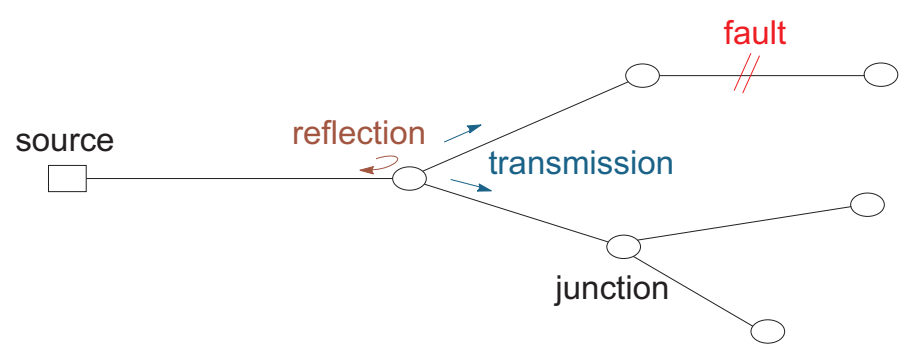

Fig. 2 Equivalent topological representation of a wire network; the transmission lines are represented with delay lines, the source with a rectangle and the discontinuities in the system with circles. The fault's position is indicated with two parallel lines.

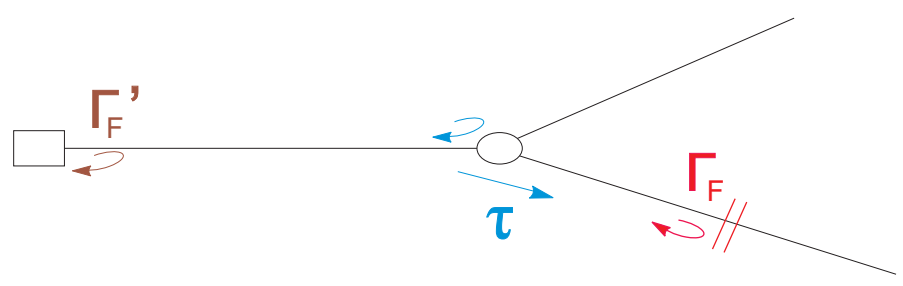

Fig. 3 Topological representation of a wire network illustrating the reflection coefficients $\Gamma_{F}^{\prime}$ and $\Gamma_{F}$.

\subsection{Equivalent topological representation}

We represented a physical model by means of a topological one, in order to highlight the elements influencing the wave propagation in the network under test, along with having a simple model to understand. We modeled the uniform lossless transmission lines with delay lines, which means that those lines are not physical objects, they only introduce time delays. We also represented the discontinuities in the system (i.e., junctions, branches, mismatched loads, etc.) with nodes, to illustrate the reflection and the transmission of the incident voltage waves. If we observe the example of Figure 1, we notice how the physical elements are modeled; furthermore, we represented, on the topological model, the reflection and transmission phenomena corresponding to the junction: when a voltage wave arrives at this position, a part is reflected back, and another is transmitted. Another example is illustrated in Figure 2, where a network is represented by means of its topological model.

A final important point to discuss in this section is the variation of the amplitude of the peaks in the received TDR echo. In fact, let us consider the example of 
Figure 3, where the fault is modeled with two parallel lines, $\tau$ is the transmission coefficient of the junction, and $\Gamma_{F}$ and $\Gamma_{F}^{\prime}$ are respectively the reflection coefficients at the fault position and the source position. We propose to illustrate how the amplitude of a certain peak changes in terms of the number of interactions with the system discontinuities. Therefore we chose a simple trajectory of the wave, which is the one from the source, through the first junction, towards the fault and then all the way back. So, if we inject a pulse into the system, its amplitude will be modified by a factor of $\tau$ when passing through the junction. This voltage wave will then reflect on the fault and its amplitude will be once more modified by a factor of $\Gamma_{F}$. The reflected wave will then follow the reverse path, and when arriving to the source position, the modification in its amplitude compared to the amplitude of the initial wave is $\tau^{2} \Gamma_{F}$. This example illustrates the idea that whenever a wave passes through several junction, its amplitude will decrease each time due to the reflection phenomena. This fact will be useful in the section 5 when analyzing mathematically the performances of the TDR and MP.

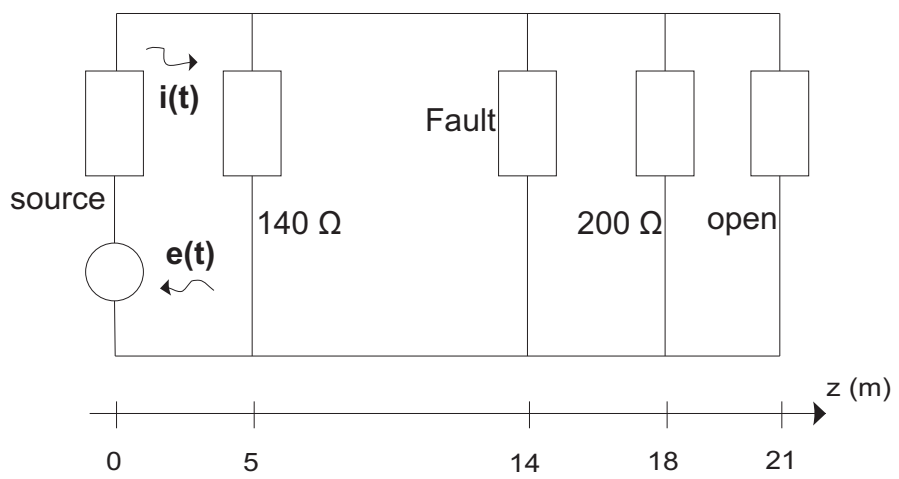

Fig. 4 An example illustrating a wire network. The fault's value is $600 \Omega$.

\subsection{Position of the network elements}

In the standard TDR case, when using the difference system, the first peak in the TDR echo we obtain corresponds to the first interaction with the fault. This idea is illustrated in the example of Figure 4. The network to be analyzed contains a fault hidden from the source by a discontinuity, at $14 \mathrm{~m}$ from the source. We consider that the fault is a parallel resistive load which value is $600 \Omega$. The echo obtained without the presence of the fault is shown in Figure 5(a), in the blue line. We notice how the different peaks correspond to the interaction of the pulse with the system discontinuities; in particular the first peak we obtain corresponds to the position 

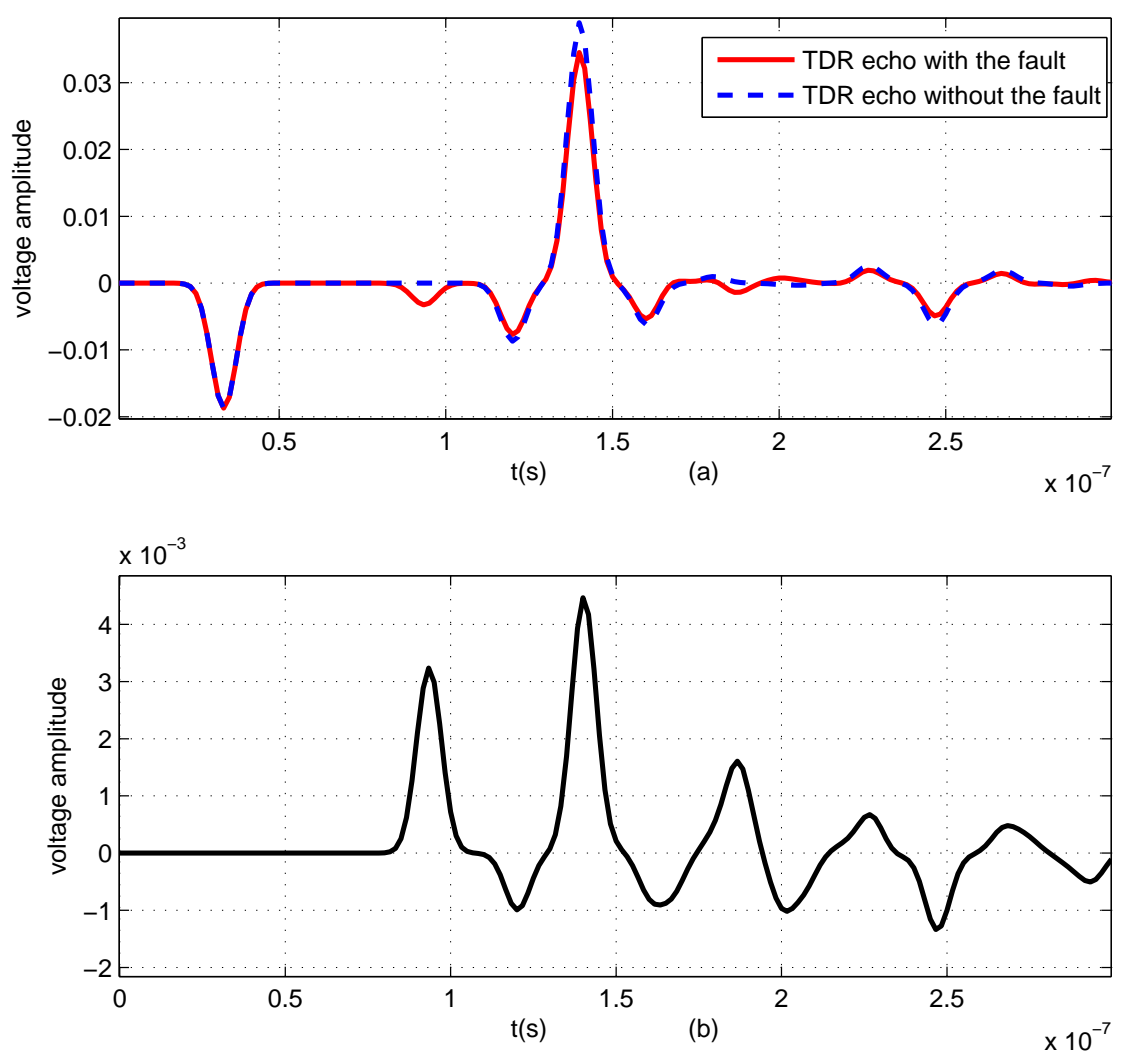

Fig. 5 (a) The reflected TDR signals in the presence and absence of the fault. (b) The difference of the two reflected signals. The studied system is illustrated in Figure 4.

of the $140 \Omega$ discontinuity (the time value on the $x$ axis is the round-trip time to the discontinuity). Furthermore, when examining the echo in the presence of the fault, we notice how its presence introduced the first variation at about $t=0.9 \mu \mathrm{s}$ from the origin, corresponding to the round-trip time to the position of this fault. Of course the peak corresponding to the $140 \Omega$ discontinuity remains unchanged, while the peaks corresponding to the discontinuities behind the fault (from the source position) are affected by the fault's presence, as we can clearly see when taking the difference signal shown in Figure 5(b). When analyzing this signal, we notice how the first variation corresponds to the fault's position, that is why we look for this peak in the detection process in TDR. Furthermore, we notice that this peak does not have the highest amplitude, as clearly shown in the figure. 
Based on this example, we can notice that the network can be divided into two main parts, regarding the fault's position: the first one upstream of the fault (between the source and the fault) and the second downstream of the fault. When examining the reflected signal in the difference system, the peaks corresponding to the first part will eventually disappear because they are the same with and without the fault (as we already noticed in the previous example); as for the peaks corresponding to the discontinuities downstream of the fault, they will be modified by the presence of the fault, thus leaving several peaks in the difference signal. Another important point is that any change in the elements upstream of the fault would affect all the peaks, including the first peak (in the difference signal), whilst any change in the elements downstream of the fault will not affect this peak.

\section{Detection gain}

After discussing the influence of different network elements on the performances of both TDR and MP, we need to find a criterion allowing us to compare those two approaches. Therefore, we propose to define a gain $G$, which we called the detection gain. Let $i(t)$ be the injected pulse in the time domain, and $e(t)$ the received signal. We note that $\alpha_{i}$ is the amplitude of the peak number $i$ in the echo of the difference system, and $t_{i}$ its position. Given that we do not have any dispersion, and also the system we are studying is a cable network, we can write the impulse response of the system as follows

$$
h(t)=\sum_{i} \alpha_{i} \delta\left(t-t_{i}\right)
$$

where $\delta(t)$ is the Dirac pulse.

Consequently, the reflected echo is

$$
e(t)=i(t) * h(t)=\sum_{i} \alpha_{i} i\left(t-t_{i}\right)
$$

Knowing that, in the detection process, we are only interested in the peak corresponding to the fault, we propose to define a gain, which we called the detection gain. Let us first calculate the energy of the received signal. It can be written as follows

$$
\mathscr{E}=\int|e(t)|^{2} d t=\int\left|\sum_{i} \alpha_{i} i\left(t-t_{i}\right)\right|^{2} d t
$$

when we calculate this expression, we find that

$$
\mathscr{E}=\sum_{i} \alpha_{i}^{2} \int i^{2}\left(t-t_{i}\right) d t+\sum_{i \neq j} \alpha_{i} \alpha_{j}^{*} \int i\left(t-t_{i}\right) i\left(t-t_{j}\right) d t
$$

If $\Delta t_{i j}=t_{j}-t_{i}$, let us denote by $\varphi\left(\Delta t_{i j}\right)$ the autocorrelation function of the injected signal 


$$
\varphi\left(\Delta t_{i j}\right)=\int i\left(t-t_{i}\right) i\left(t-t_{j}\right) d t=\int i(s) i\left(s-t_{j}+t_{i}\right) d s
$$

In the TDR case, we assume that the injected pulse is normalized in terms of its energy. The energy of the echo can thus be written as

$$
\mathscr{E}=\sum_{i} \alpha_{i}^{2}+\sum_{i \neq j} \alpha_{i} \alpha_{j}^{*} \varphi\left(\Delta t_{i j}\right)
$$

We know that the coefficients $\alpha_{i}$ are influenced by the discontinuities in the system (as discussed in section 5); their values decrease according to the number of interactions with the system discontinuities (the evolution of these values is a geometric series).

We also note that Equation (7) is composed of two terms: the first one is the average energy of the signal $\sum_{i} \alpha_{i}^{2}$, and the second one is the mutual energy of the peaks of this signal $\sum_{i \neq j} \alpha_{i} \alpha_{j}^{*} \varphi\left(\Delta t_{i j}\right)$.

When examining the expression of the mutual energy, we can notice that it contains two important parts: the first one $\alpha_{i} \alpha_{j}^{*}$ which depends on the number of interactions, and the second one is the correlation function $\varphi\left(\Delta t_{i j}\right)$. If the temporal support of this function is narrow, then this expression can be neglected and the dominant part would be that of the average energy. In fact, we know that the first peaks we observe have the highest amplitude, and when injecting a pulse into the system, those peaks are separated one from another. We also know that the peaks appearing after those first peaks (in time) would have small amplitudes and the mutual energy between them can be neglected. So, we can reasonably assume that

$$
\sum_{i \neq j} \alpha_{i} \alpha_{j}^{*} \varphi\left(\Delta t_{i j}\right) \ll \sum_{i} \alpha_{i}^{2}
$$

In this case, the energy of the first peak (the one we consider when detecting the fault) would be

$$
\mathscr{E}_{\mathrm{TDR}}^{F}=\alpha_{1}^{2}
$$

In the MP case, the same procedure is applied, but here the injected signal is the time reversed version of the TDR echo. The MP echo is written as

$$
e(t)=i(t) * h(t) * h(-t)
$$

Defining $g(t)=h(t) * h(-t)$, we can write

$$
g(t)=\sum_{k} \beta_{k} \delta\left(t-t_{k}^{\prime}\right)
$$

where $\beta_{i}$ is the amplitude of the peak number $i$. In fact, if we calculate $g(t)$ in terms of $\alpha_{i}, t_{j}$ and $t_{i}$ we find

$$
g(t)=\sum_{i} \sum_{j} \alpha_{i} \alpha_{j} \delta\left(t+t_{j}-t_{i}\right)
$$


Comparing Equations (11) and (12) we find that

$$
\beta_{k}=\sum_{i, j}^{k=j-i} \alpha_{i} \alpha_{j}
$$

and also

$$
t_{k}^{\prime}=t_{j}-t_{i}
$$

Note that when we time-reverse the TDR echo to obtain the injected signal in the MP case, we are effectively doing a time shifting operation (i.e., $g(t)=h(t) *$ $\left.h\left(t_{1}-t\right)\right)$. The value of the peak at $t=0$ corresponds to the fault; this peak has an amplitude equal to the average energy of the injected signal, that is the TDR echo in this case. Under the same assumption that the initial pulse (i.e., the injected pulse in the TDR) is normalized in energy, the energy of the MP peak is

$$
\mathscr{E}_{\mathrm{MP}}^{F}=\left|\sum_{i} \alpha_{i}^{2}\right|^{2}
$$

Let $\mathscr{E}$ TDR and $\mathscr{E}_{\mathrm{MP}}$ be respectively the energies of the testing signals in the TDR and MP cases. In order to be able to compare the TDR and MP approaches, we need to have the same injected energy. That is why we propose to normalize according to the injected energy in both cases. The detection gain $G$ can be thus defined as the ratio of the normalized energy of the MP peak to the normalized energy of the TDR peak

$$
G=\frac{\mathscr{E}_{\mathrm{MP}} / \mathscr{E}_{\mathrm{MP}}}{\mathscr{E}_{\mathrm{TDR}} / \mathscr{E}_{\mathrm{TDR}}}
$$

Based on the previous analysis, when the different peaks do not interfere one with another, $G$ can be expressed as follows

$$
G=\frac{\sum_{i=1}^{\infty} \alpha_{i}^{2}}{\alpha_{1}^{2}}
$$

We can clearly notice that $G \geq 1$, and consequently the MP approach always presents an advantage over the TDR; this advantage is greater in the case where we have multiple peaks, resulting in a higher gain. In practice, we consider the first $N$ peaks when calculating the gain, so as to have

$$
G \approx \frac{\sum_{i=1}^{N} \alpha_{i}^{2}}{\alpha_{1}^{2}}
$$

In the next section we propose to illustrate the previously discussed ideas through simulation results. 


\section{Simulation results}

\subsection{Analyzed configurations}

We consider the configurations illustrated in Figures 6 and 7, where the fault is in front of the source in the first case, then masked from the source by several discontinuities in the second case. We chose those configurations because they represent two extreme cases; thus it seems interesting to compare the performances of the MP and TDR in both cases.

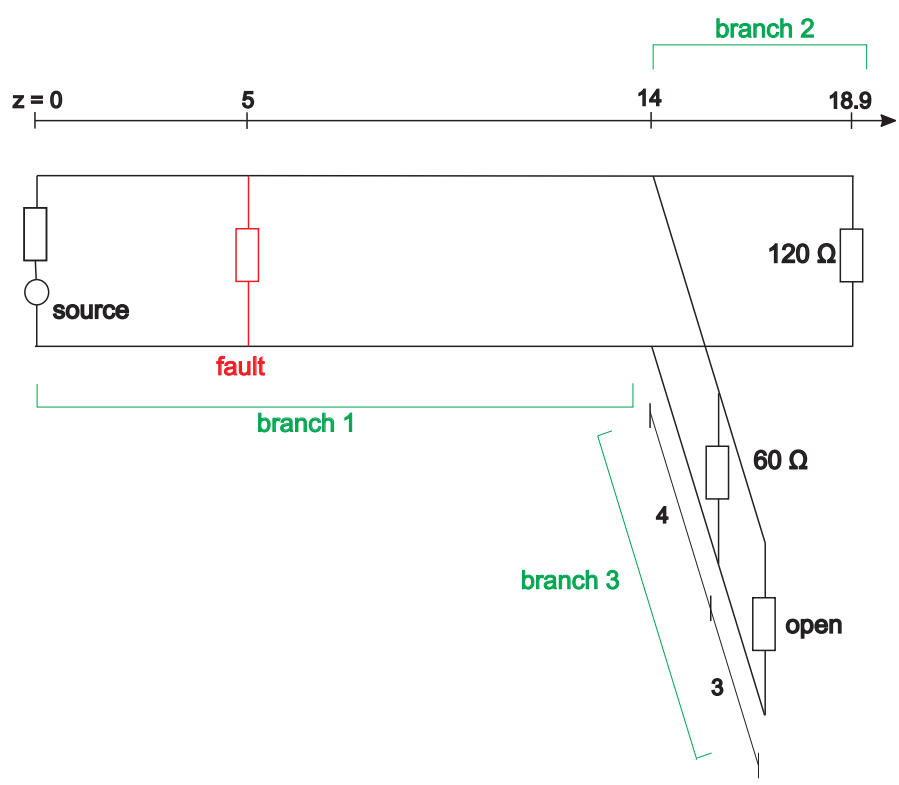

Fig. 6 The analyzed network in the first case, where the fault is in front of the source. All lengths are in meters.

\subsection{Numerical results}

We simulated the voltage propagation in the configurations of Figures 6 and 7 using transmission line theory as presented in [8]. The characteristic impedance of the lines is chosen to be $75 \Omega$ (such as for some coaxial cables). We will verify the obtained values of the detection gain according to the general formula (Equation 16) and the simplified formula (Equation 18). In this last case, we will be examining a 


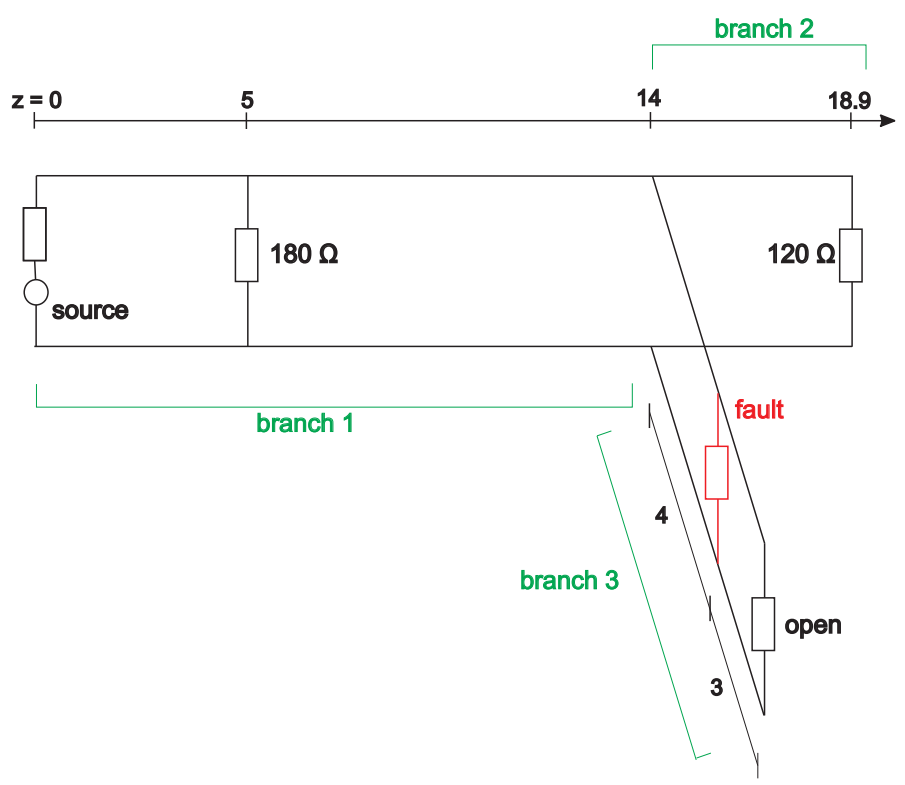

Fig. 7 The analyzed network in the second case, where the fault is embedded in the system. All lengths are in meters.

limited number of peaks, thus specifying an inferior limit to the gain (referred to as a threshold in the Table 1).

Table 1 Numerical results obtained for the configuration of Figure 7.

\begin{tabular}{|c|c|c|}
\hline Fault value $(\Omega)$ & Predicted gain & ulated gain \\
\hline Short & 2.33 & 2.6 \\
\hline 30 & 3.4 & 4 \\
\hline 600 & 7.7 & 9.22 \\
\hline
\end{tabular}

In the first case (Figure 6), we chose a value of the fault equal to $600 \Omega$, corresponding to a soft fault. The inferior limit of $G$ in this case is 1.55 (we considered $\mathrm{N}=5$ ). When we calculate its exact value, we find $G=1.73$. In the second case (Figure 7) when the fault is masked from the source by several discontinuities, we chose several values of the fault, illustrated in Table 1. In this configuration, the fault is separated from the source by a discontinuity at $5 \mathrm{~m}$ and a junction at $14 \mathrm{~m}$. The numerical values obtained when calculating the inferior limit of the detection gain, along with its exact values are illustrated in Table 1. When examining those results, we notice that the more the fault is soft (i.e., the reflection coefficient at the source position is small), the more the MP becomes effective when compared to standard TDR. If we also compared the two studied configurations (Figures 6 and 7), when 
the fault's value is $600 \Omega$, we notice that when the fault is embedded in the system, the gain's value is greater than the case where the fault is directly in front of the source. In fact, in the first case we have a greater number of peaks than in the second case; so as predicted by Equation 18, we have a greater value of the detection gain.

\section{Conclusion}

In this paper, we evaluated the impact of the network topology on the performance of the TDR and MP approaches. A topological study allowed us first to state what are the most influencing factors on the effectiveness of the TDR, then a mathematical analysis proved the advantage of the MP method over the standard TDR. The discussed ideas were finally verified through simulation results. This whole study allowed a better understanding of the factors influencing the TDR performance, thus enabling, for any configuration, to predict the effectiveness of the MP approach compared to standard TDR.

\section{References}

1. Furse S, and Haupt R (2001) Down to the wire. IEEE Spectrum 38:34-39

2. Review of federal programs for wire system safety. National Science and technology Council, White House, Final Report, 2000

3. Furse C, Chung Y.C., Dangol R., Mabey M.N.G. and Woodward R. (2003) Frequency domain reflectometry for on-board testing of aging aircraft wiring. IEEE Transactions on Electromagnetic Compatibility 45:306-315

4. Smith P, Furse C. and Gunther J. (2005) Analysis of spread spectrum time domain reflectometry for wire fault location. IEEE Sensors Journal 5:1469-1478

5. Abboud L., Cozza A. and Pichon L. (2009) Utilization of matched pulses to improve fault detection in wire networks. 9th international conference on ITS Telecommunications

6. Fink M. (1992) Time Reversal of Ultrasonic fields - part 1: basic principles. IEEE Transactions on Ultrasonics, Ferroelectrics and frequency control 39:555-566

7. Papoulis A. (1977) Signal Analysis. In: Nalle P. and Gardner M.(ed), McGraw-Hill.

8. Paul C.R. (1994) Analysis of multiconductor transmission lines. In: Chang K.(ed), WileyInterscience. 